\title{
Intermédialités
}

Histoire et théorie des arts, des lettres et des techniques

Intermediality

History and Theory of the Arts, Literature and Technologies

\section{Film-théâtre, intermédialité et nouveaux enjeux esthétiques}

\section{Sandrine Siméon}

Numéro 33, printemps 2019

restituer (le temps)

rendering (time)

URI : https://id.erudit.org/iderudit/1065016ar

DOI : https://doi.org/10.7202/1065016ar

Aller au sommaire du numéro

Éditeur(s)

Revue intermédialités

ISSN

1920-3136 (numérique)

Découvrir la revue

Citer cet article

Siméon, S. (2019). Film-théâtre, intermédialité et nouveaux enjeux esthétiques. Intermédialités / Intermediality, (33). https://doi.org/10.7202/1065016ar
Résumé de l'article

Comment l'analyse intermédiale permet-elle de souligner l'interpénétration médiatique à l'oeuvre entre la performance scénique et les modes d'écriture de l'enregistrement audiovisuel du spectacle vivant ? Cet article évalue les manières dont la rhétorique filmique agit sur le dispositif scénique, puis comment, à son tour, la scène influence l'esthétique inédite du film-théâtre, lui conférant un statut autotélique. 


\title{
Film-théâtre, intermédialité et nouveaux enjeux esthétiques
}

\author{
SANDRINE SiMÉon
}

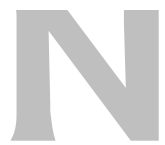

ombre de spécialistes ont tenté de définir le processus à l'œuvre lors de l'enregistrement audiovisuel d'une performance scénique. Pourtant, les publications sur les captations et les enregistrements audiovisuels se limitent souvent à des études de cas ponctuelles et abordent en majeure partie ce qui se passe sur scène, ne réservant aux spécificités proprement filmiques, à ce qui se passe sur l'écran, que peu de réflexions théoriques.

En effet, jusque dans les années i990, la réflexion concernant le filmage du théâtre se réduisait à l'article fondateur d'André Bazin, sur lequel se sont ultérieurement greffées quelques remarques périphériques. Bazin n’est pas le premier à considérer l'éventail des relations entre théâtre et cinéma - Meyerhold avait déjà considéré leurs interactions dès les années 1920 - mais son essai de 1951, « Théâtre et cinéma I », fait école en ce qu'il tente de réhabiliter un mode d'écriture qui est traditionnellement controversé. La transposition de textes dramatiques et spectaculaires à l'écran est une pratique jusqu'alors mal acceptée, désignée sous l'expression réductible de «théâtre filmé ». Bazin propose alors de remplacer cette expression trop empreinte de négativité par « théâtre cinématographique ${ }^{2} »$.

Depuis, Patrice Pavis avait traité de manière succincte, dans une infime partie de son ouvrage de 1996, L'analyse des spectacles, les statuts de la caméra, de l'acteur et de la scénographie, à l'aide d'un film en particulier, Marat-Sade, de Peter Brook

\footnotetext{
I André Bazin, «Théâtre et cinéma », Esprit, n 19, juin 1951, p. 232-253, https://esprit.presse.fr/article/bazin-andre/theatre-et-cinema-25576 (consulté le 20 mars 2019).

${ }^{2}$ Ibid., p. 25 I.
} 
(1967). André Helbo avait publié en 1997 une monographie d'inspiration sémiologique, L'adaptation: du théâtre au cinéma, qui passait en revue les formes de relations possibles entre théâtre et cinéma, sans toutefois interroger les spécificités de tels enregistrements. En 2007, l'ouvrage très complet de Pascal Bouchez, Filmer l'éphémère: récrire le théatre (et Mesguich) en images et en sons, ne porte toutefois qu'exclusivement sur les expériences d'enregistrement des productions de Daniel Mesguich. Certaines revues comme la Revue d'Histoire du Théâtre et Théâtre Aujourd'bui ont depuis consacré quelques articles ou numéros au filmage du théâtre; des colloques et journées d'étude ont aussi publié certaines de leurs communications ou de leurs actes. Enfin, deux publications sur le théâtre d'Ariane Mnouchkine ont récemment vu le jour. Dans L'art du présent (2016), de Fabienne Pascaud, aucun de ses entretiens avec Mnouchkine ne mentionne l'influence de la caméra sur son travail de metteuse en scène - son travail de cinéaste, pourtant manifeste, semblant ainsi se limiter à son film biographique sur Molière. De même, la publication de Béatrice Picon-Vallin intitulée Le Théâtre du Soleil. Les cinquante premières années (2014) est un récit historique sur la troupe du Théâtre qui ne signale le travail de Mnouchkine avec ce médium que sur deux pages, alors que Mnouchkine, intéressée par le dialogue entre la scène et l'écran a, depuis $1789^{3}$, exploré les diverses possibilités de mise en images de ses créations scéniques - c'est à partir de ce moment que le film a fortement marqué une nouvelle étape pour la troupe, sans compter qu'il a aussi permis de pérenniser son répertoire.

Un ouvrage en particulier s'est pourtant distingué en 1997. Le film de théâtre ${ }^{4}$, de Béatrice Picon-Vallin, réunit les témoignages de praticiens du théâtre et du cinéma sur leurs approches lors du passage de la scène à l'écran, examine l'existence $\mathrm{du}$ « film de théâtre » comme genre à part entière et présente quelques modalités de traitement du théâtre par le cinéma. Picon-Vallin constate déjà la vaste variété des formules désignant cette pratique et suggère l'expression « film de théâtre » qui, à son sens, reste la moins péjoratives.

Néanmoins, si à partir de la fin des années 1990, les publications sur le sujet dénotent une amorce d'intérêt pour cette pratique audiovisuelle, elles demeurent, somme toute, exceptionnelles, et n'abordent que rarement ce qui distingue conceptuellement ce type de film du reste de la production filmique. D'autant plus

\footnotetext{
${ }^{3}$ Ariane Mnouchkine, 1789 , BelAir Classique, 2017.

${ }_{4}$ Béatrice Picon-Vallin (dir.), Le film de théatre, Paris, CNRS éditions, coll. « Arts du spectacle » (dirigée par Elie Konigson), 1997.

5 Béatrice Picon-Vallin, 1997, p. Io.
} 
que chaque spécialiste, s'étant donné la peine d'y réfléchir, propose de nouveaux concepts, souvent afférents à leur propre domaine de recherche.

Le « film de théâtre » continue en effet de renvoyer à une notion vague qui rassemble tout le théâtre filmé sans claire distinction entre, par exemple, un spectacle capté pour sa transmission simultanée ou en différé en salles, sur les chaînes télévisées ou sur des plateformes culturelles numériques; une interprétation scénique filmée avec ou sans public pour son édition en DVD; et l'adaptation cinématographique d'un texte dramatique.

En nommant «film[s]-théâtre[s] ${ }^{6} \gg$ les films produits d'après l'enregistrement de performances scéniques, j'ai proposé d'ouvrir une catégorie particulière en mesure d'accueillir tous films, qu'ils soient tournés en la présence ou l'absence de public, en synchronie avec une présentation effective, ou encore selon ses fonctions, pourvu qu'ils exhibent deux propriétés distinctives: une énonciation affranchie de celle du spectacle et la valorisation du point de vue du public de l'écran. Seules ces deux caractéristiques me paraissent essentielles à la mise en valeur des qualités propres au film-théâtre.

Après avoir considéré diverses approches, allant des théories sur la traduction de Walter Benjamin 7 à l'intertextualité d'après Julia Kristeva ${ }^{8}$, en passant par les phénomènes intersémiotiques avec André Helbo ${ }^{9}$, l'adaptation selon Linda Hutcheon ${ }^{\mathrm{IO}}$ et les considérations intermédiales de chercheurs comme Jürgen $\mathrm{E}$.

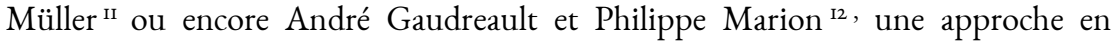
particulier m'a permis de conceptualiser le film-théâtre et de confirmer sa raison d'être en tant qu'événement intermédial.

${ }^{6}$ Sandrine Siméon, «Le film-théâtre, un troisième temps du théâtre ? », Agathe Torti Alcayaga et Christine Kiehl (dir.), Théâtre, levain du cinéma. Théâtre, destin du cinéma, Paris, Le Manuscrit, 2013, p. 169-183.

7 Walter Benjamin, « The Task of the Translator. An Introduction to the Translation of Baudelaire's Tableaux Parisiens $\gg$, Illuminations: Essays and Translations, Hannah Harendt (dir.), trad. Harry Zohn, New York, Schocken Books, 1968, p. 69-82.

${ }^{8}$ Julia Kristeva, Semeiotike. Recherches pour une sémanalyse, Paris, Seuil, 1969, p. 143-173.

9 André Helbo, L'adaptation. Du théatre au cinéma, Paris, Armand Colin, 1997.

${ }^{10}$ Linda Hutcheon, $A$ Theory of Adaptation, $2_{2}^{\mathrm{e}}$ édition, New York, Routledge, 2013.

II Jürgen E. Müller, «L'intermédialité, une nouvelle approche interdisciplinaire: perspectives théoriques et pratiques à l'exemple de la vision de la télévision », Cinémas, vol. ı, n²-3, 2000, p. I05-134, https://id.erudit.org/iderudit/024818ar (consulté le I6 mars 2019).

${ }^{12}$ André Gaudreault et Philippe Marion, « The Cinema as a Model for the Genealogy of Media », Convergence, vol. 8, no 4, 2002, p. I2-18, https://doi.org/Io.II77\%2Fr35485650200800402 (consulté le I6 mars 2019). 
Irina Rajewsky, dans son article, «Intermediality, Intertextuality, and Remediation: A Literary Perspective on Intermediality ${ }^{13} \gg$, propose en effet de nouvelles possibilités de réflexions sur les phénomènes d'hybridation. Par exemple, Rajewsky estime l'intertextualité inappropriée pour décrire les transferts entre les médias, puisque celle-ci « is understood in the limited sense of references by a (literary) text either to individual other texts or to literary (sub)systems ${ }^{14} \gg$. Selon elle, l'intertextualité appartient à une sous-catégorie de références intermédiales, alors que l'intermédialité implique plus spécifiquement « a crossing of media borders, and thus a medial difference ${ }^{\mathrm{Is}} \gg$.

À l'instar du débat autour de l'intertextualité qui a servi de fondation à la théorisation de l'intermédialité dans les années 1970, Linda Hutcheon, avec $A$ Theory of Adaptation, propose de réunir les transferts médiatiques sous la catégorie unique d'adaptations sans distinguer leurs spécificités respectives ${ }^{16}$, envisageant ces adaptations comme des « re-mediations, that is, specifically translations in the form of intersemiotic transpositions from one sign system (for example, words) to another (for example, images). This is translation but in a very specific sense: as transmutation or transcoding, that is, as necessarily a recoding into a new set of conventions as well as signs 17 ». Cette définition aurait pu servir de point de départ pour explorer les modi operandi du film-théâtre et considérer que filmer une performance revient à passer d'un système de signes, scénique, à un autre, filmique. Pourtant, considérer ces films comme des adaptations minimise leurs qualités intermédiales et menace l'hétérogénéité des échanges avec la scène, qui contribuent à l'esthétique singulière du film-théâtre.

En revanche, l'approche de Rajewsky offre une interprétation plus nuancée de la multiplicité des transferts intermédiatiques et des manières dont opèrent les

13 Irina O. Rajewsky, « Intermediality, Intertextuality, and Remediation: A Literary Perspective on Intermediality », Intermédialités, no 6, automne 2005, p. 43-64, https://id.erudit.org/iderudit/Ioos505ar (consulté le I6 mars 2019).

${ }^{14}$ Ibid., p. 54.

Is Ibid.

${ }^{16}$ Afin de différencier les diverses dimensions de l'adaptation, Linda Hutcheon propose une double définition de l'adaptation: comme produit, «as extensive, particular transcoding », et comme processus, «as creative reinterpretation and palimpsestic intertextuality $\gg$. Hutcheon, 20I3, p. 22.

${ }_{17}$ Ibid., p. 16. 
connexions entre différents médias afin de souligner leur influence réciproque. En effet, non seulement comprend-elle l'intermédialité comme

a generic term for all those phenomena that (as indicated by the prefix inter) in some way take place between media. "Intermedial” therefore designates those configurations which have to do with a crossing of borders between media, and which thereby can be differentiated from intramedial phenomena as well as transmedial phenomena (i.e., the appearance of a certain motif, aesthetic, or discourse across a variety of different $\left.\operatorname{media}^{18}\right)$.

Mais surtout, Rajewsky reconnaît que les divers transferts entre médias nécessitent leur propre désignation. Ainsi, au lieu de développer une catégorie unique, elle propose trois sous-catégories d'intermédialité, dont celle qu'elle surnomme 《références intermédiales ${ }^{19}$ », décrivant les manières dont un média utilise ses propres moyens d'expression pour faire référence à un autre média. Cette configuration implique deux médias distincts, mais seul l'un des deux est matériellement présent. Dans le cas du film-théâtre, issu d'un enregistrement audiovisuel de la scène, le film est le support matériellement présent, le média de référence qui se constitue en relation avec le média (et ses spécificités) auquel il réfere, en l'occurrence le spectacle qu'il filme: « rather than combining different medial forms of articulation, the given media-product thematizes, evokes, or imitates elements or structures of another conventionally distinct medium through the use of its own media-specific means ${ }^{20} \gg$. Cette catégorie de « références intermédiales 》 décrit donc plus précisément les processus de production de sens du film-théâtre et l'influence des échanges syncrétiques entre la scène et l'écran sur son esthétique, tout en acceptant un éventail plus large d'événements scéniques que les notions établies précédemment - captations de performances en public pour une retransmission en direct ou ultérieure, enregistrements de spectacles plus tard modifiés en vue d'une édition DVD ou encore d'un visionnement sur des plateformes Web.

Afin de mieux comprendre comment se concrétisent ces références intermédiales et de quelles manières elles agissent sur l'esthétique du film-théâtre, je propose d'examiner certaines conventions de mise en scène que celui-ci ajuste à ses propres codes. Je présenterai toutefois d'abord les procédés filmiques qu'adoptent les praticiens de théâtre dans la conception de leurs dispositifs scéniques afin d'enrichir

\footnotetext{
${ }^{18}$ Rajewsky, 2005, p. 46.

${ }^{19} \mathrm{Ma}$ traduction.

${ }^{20}$ Rajewsky, 2005, p. 53.
} 
la portée de leur mise en scène ou d'amplifier le point de vue du public de théâtre, puisque les arts de la scène évoluent désormais aux côtés des arts filmiques et que cette relation intermédiale est mutuellement enrichissante. Enfin, si cette interdépendance détermine l'esthétique du film-théâtre, il me semble nécessaire d'ajouter quelques remarques quant à son statut et de l'évaluer à la lumière de la tension entre « document » et « monument » divulguée par Michel Foucault.

Dès le début $\mathrm{du} 2 \mathrm{O}^{\mathrm{e}}$ siècle, les praticiens du théâtre ont entretenu des liens avec le cinéma: intégration d'écrans au dispositif scénique ${ }^{2 \mathrm{I}}$, enregistrement de performances $^{22}$ ou diffusion simultanée de captations ${ }^{23}$, appropriation d'artifices cinématographiques. L’expérience réalisée par André Engel ne déroge pas à ce penchant et témoigne des manières dont la rhétorique filmique stimule la mise en scène contemporaine. Engel présente Woyzeck, de Georg Büchner, au Centre dramatique national de Savoie ${ }^{24}$ en 1998 . Le dispositif scénique est formé de quatre cadres mobiles - l'envers de ces espaces est également aménagé en décors - qui permettent les changements de lieu. À chaque cadre correspond une action singulière se déroulant dans un espace-temps unique - structure similaire à celle d'une scène de film - , chaque changement de cadre évoquant une coupure au montage. En effet,

${ }^{21}$ Parmi les pionniers de l'utilisation des écrans sur scène, Meyerhold et Eisenstein ont également contribué aux réflexions théoriques concernant les échanges entre théâtre et cinéma. Béatrice Picon-Vallin (dir.), Les écrans sur la scène : tentations et résistances de la scène face aux images, Lausanne, L'Âge D'Homme, coll. « Théâtre XX' siècle », 1998.

${ }^{22}$ La Comédie-Française s'était, dès 1908, associée avec la société « Le film d'art », fondée par les frères Lafitte afin de promouvoir le cinéma, alors considéré comme un spectacle populaire. Outre le fait que les rôles des acteurs sont tenus par d'importants sociétaires, L'assassinat du Duc de Guise (André Calmettes et Charles le Bargy, 1908) marque un tournant dans l'histoire du cinéma et le début d'une importante coopération. Les premières captations scéniques en France remontent également au début du $20^{\mathrm{e}}$ siècle. Anticipant les possibilités de propagation des productions de la troupe, Émile Fabre, alors administrateur de la ComédieFrançaise, initia les premiers tournages de spectacles dans un studio installé depuis 1932 dans l'enceinte du ministère des Postes, Télégraphes et Téléphones. La Comédie-Française inaugure le premier enregistrement audiovisuel d'un de ses spectacles, Les précieuses ridicules, en 1935. Au début du siècle, Méliès avait également filmé ses performances, enrichissant ses tours de magie d'effets spéciaux permis par la technique filmique.

${ }^{23}$ À l'instar du National Theater Live de Londres, qui diffuse ses productions dans plus de 700 salles d'une vingtaine de pays, Éric Ruf, administrateur de la Comédie-Française, a signé un partenariat de trois ans avec le groupe Pathé, pour la diffusion de trois spectacles retransmis en direct de la salle Richelieu dans 300 salles en France et à l'étranger à partir de l'automne 2016, http://www.pathelive.com/programme/comedie-francaise\#programme (consulté le 20 mars 2019).

${ }^{24}$ Engel avait déjà présenté une version de Woyzeck sous le titre La nuit des chasseurs en I988 au Théâtre national de la Colline. 
durant les manipulations de cette structure amovible, la scène s'obscurcit, rappelant les noirs entre les images de la pellicule d'un film. Si un tel dispositif s'avère ludique, en ajustant son interprétation selon des procédés filmiques, Engel stimule en même temps son écriture scénique. La nature du récit de Woyzeck, le reportage - caractérisé par son aspect fragmentaire - , détermine le choix structural d'Engel auquel le modèle du découpage séquentiel et du montage correspond précisément : une série de faits qui se succèdent, construisant la fiction à la manière d'une suite de scènes de film, chaque cadre correspondant à l'une d'elles.

De manière similaire, lors de sa mise en scène des Éphémères en 2006, Ariane Mnouchkine adopte un dispositif scénique qui correspond à l'exigence narrative de son spectacle, composé d'une multiplicité de vignettes. Les décors, par conséquent extrêmement nombreux, sont simplifiés afin de permettre une vaste mobilité spatiotemporelle. L'essence de chaque décor est ainsi disposée sur des plateaux mobiles, ou chariots, manipulés par des machinistes qui facilitent leurs entrées et sorties de la scène. Ce dispositif (voir la figure i) s'accorde précisément avec le motif du mouvement qui imprègne la totalité rythmique du spectacle - chaque chariot entre et quitte lentement la scène selon une trajectoire latérale dans un constant mouvement de rotation.

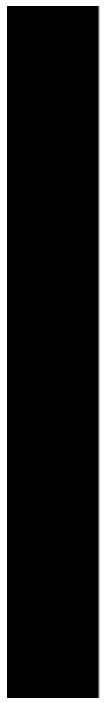

\section{Les Ephémères : topographie du dispositif scénique}

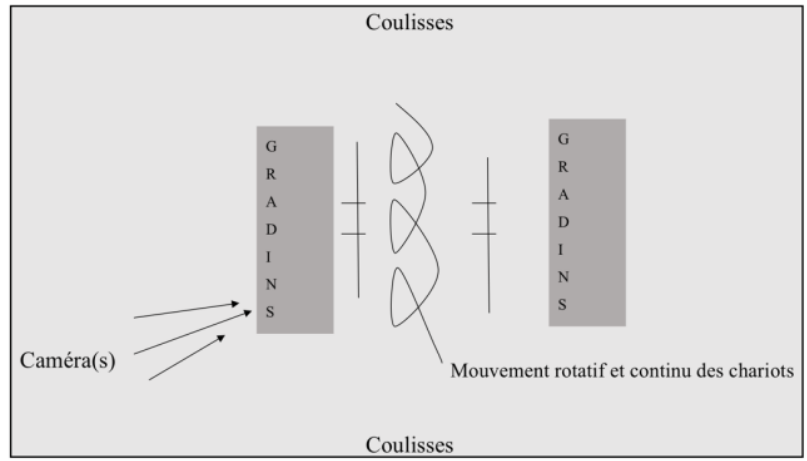

Figure I. Topographie de l'espace scénique. (C) Sandrine Siméon.

Cette rotation des chariots garantit au public de la salle une perspective démultipliée et sans cesse renouvelée qui lui est habituellement interdite; ubiquité qui, par ailleurs, se rapproche de celle à laquelle le public de l'écran est habitué. 
Pourtant, alors qu'esthétiquement, comme dans la matière de sa fable, Les éphémères refusent la fixité, lors du tournage du film, la caméra est relativement stable — ses mouvements sont surtout de nature optique et comprennent d'occasionnels courts panoramiques, mais aucun travelling. Or, le dispositif scénique élaboré par Mnouchkine masque cette stasis de manière originale, puisque les effets de mouvement sont créés de manière extrinsèque au film. Ainsi, lors de scènes d'action - des ambulanciers essaient de raviver une femme, une mère et sa petite fille dansent à la cadence d'une musique rapide —, ce ne sont ni les mouvements de caméra ni le montage dont le rythme s'accélère, mais plutôt les chariots qui pivotent plus rapidement. De même, simulant un arrêt sur image, un chariot stoppe brusquement lorsqu'un homme, en train de dîner avec sa femme, s'effondre sur son assiette. Les personnages restent immobiles le temps d'une rotation du plateau qui stoppe à nouveau. La femme se réanime alors que le chariot reprend le cours de ses lentes révolutions, dans le sens inverse cette fois. Mnouchkine explore ainsi les moyens de dynamiser l'espace scénique des Éphémères et de multiplier les possibilités focalisatrices de son public grâce à des moyens qu'elle emprunte à l'esthétique filmique et qu'elle ajuste à la scène : le mouvement des plateaux qui pivotent sur leur axe produit un effet similaire à celui d'une caméra qui tournerait autour des décors sans que pourtant la caméra n'intervienne sur la scène. Le dispositif scénique compense ainsi les contraintes de tournage, puisque la mobilité des chariots masque l'immobilité des caméras - pendant la performance en public, les caméras sont disposées de manière à ne pas le gêner et leurs mouvements ont tendance à être contraints.

Si les mises en scène d'Engel et de Mnouchkine empruntent aux conventions filmiques pour transcender les limitations scéniques, satisfaire les exigences spatiotemporelles du récit, ou rivaliser avec l'ubiquité souvent réservée aux arts visuels, cet « intercannibalisme $e^{25}$ » médiatique peut également renouveler la lecture d'interprétations scéniques.

Par exemple, la mise en scène initiale de La cantatrice chauve par Nicolas Bataille en I95I avait respecté les didascalies descriptives d'Eugène Ionesco prescrivant un intérieur bourgeois (anglais). En revanche, celle de Jean-Luc Lagarce en $199 \mathrm{I}^{26}$ met

${ }_{25}$ Terme utilisé par Patrice Pavis dans L'analyse des spectacles : théâtre, mime, danse, dansethéâtre, cinéma, Paris, Nathan, 1996, p. 299.

${ }^{26}$ Cette mise en scène sera reprise à l'identique par François Berreur en 2006, puis filmée par Vincent Bataillon en 2007. 
en scène des éléments non préconisés dans le texte dramatique. Afin de rendre compte de l'insolite du théâtre de Ionesco, et de suggérer un monde de non-sens, factice et stéréotypé, Lagarce avait opté pour un décor kitsch ${ }^{27}$, s'inspirant de ceux, artificiels, des sitcoms : couleurs saturées, faux gazon, façade de maison en carton-pâte, éclairages. Il exploite encore d'autres éléments télévisuels aisément reconnaissables : fondus au noir censés figurer les interruptions publicitaires, pauses dans les répliques des personnages permettant aux rires préenregistrés de se faire entendre. Lagarce modernise ainsi l'interprétation de La cantatrice chauve, l'ajustant au contexte des années 1990. Alors que le texte de Ionesco parodiait une certaine faillite de la communication interpersonnelle pour mieux la dénoncer, cette incommunicabilité est réinterprétée par Lagarce à l'aide d'éléments télévisuels qu'il greffe à sa mise en scène, incohérence que s'appropriera ensuite Vincent Bataillon lorsqu'il filmera cette mise en scène en 2007. En effet, à son tour, la réalisation de Bataillon viendra incarner ces attributs outranciers en aménageant une passerelle entre la rhétorique filmique, les spécificités scéniques et les thématiques du texte dramatique. Bataillon multiplie ainsi les plans immotivés du diamant au doigt de Madame Smith et de ses escarpins vernis rose vif. Il démultiplie également les prises de vue témoignant des réactions des personnages aux répliques de leurs homologues afin de brouiller les perspectives : à qui appartiennent ces points de vue ? L'arbitraire et l'incertain deviennent alors garants de la cohérence du filmage, tout en illustrant les thèmes de l'absurde inspirés des interprétations précédentes. Cet exemple est d'autant plus intéressant que les thèmes de l'absurde sont mis en abyme par le truchement de trois médias hétérogènes.

De même que ces trois spectacles exploitent les ressources de l'illusion filmique tout en opérant sur le terrain du théâtre, les réalisateurs exploitent à leur tour les conventions scéniques, les ajustant à la rhétorique du film-théâtre pour mieux le distinguer de ses homologues - films de fiction et documentaires.

Pour la réalisation de son film Le bal, en 1983 , d'abord mis en scène par JeanClaude Penchenat deux ans plus tôt, Ettore Scola ne tourne pas sur la scène d'origine ${ }^{28}$ et modifie la distribution initiale en y ajoutant, entre autres, Penchenat. Concernant ses stratégies de filmage, Scola privilégie certains procédés cinématographiques, en l'occurrence le plan large et le plan-séquence. Le plan large, ou plan d'ensemble, à vocation descriptive, permet de situer les personnages dans leur environnement

\footnotetext{
${ }^{77}$ Dans le supplément du DVD, Yannic Mancel (conseiller artistique du Théâtre du Nord) rappelle que Lagarce avait voulu suggérer avec un tel décor que le théâtre est un jeu, qu'il reste une activité ludique en décalage avec le monde réel.

${ }^{28}$ Le Théâtre Firmin Gémier/La Piscine, Antony, France.
} 
spatial, tandis que le plan-séquence présente une suite d'images sans coupures au montage et restitue l'action dans son unicité temporelle. Lorsque, pour le tournage du Bal, Ettore Scola favorise ces deux procédés filmiques, il évoque l'ensemble spatiotemporel constitué auparavant par le texte scénique dont il s'inspire. Il fait ainsi émerger des liens esthétiques d'interdépendance entre deux modes de présentation distincts et met donc au jour, corollairement, l'intermédialité inhérente à l'énonciation du film-théâtre.

De manière similaire, dans Le dernier caravansérail, film de Mnouchkine d'après sa mise en scène du même nom (2006), une prise de vue cadre une main écrivant ce que relate une voix hors champ. Des personnages sont visibles en surimpression sur le plateau de la Cartoucherie, où, durant les jonctions entre deux tableaux du spectacle, un changement de décor s'exécute à la vue du public de la salle. Ces moments, inhérents aux spectacles de Mnouchkine, sont donc ici mis en scène par l'intermédiaire d'un procédé filmique, la surimpression, qui dynamise l'action relativement statique de l'écriture, tout en réintroduisant le processus théâtral. Mnouchkine ne cherche effectivement pas à effacer la machinerie du théâtre; au contraire, elle enrichit ainsi ses films de moments souvent occultés lors du montage en postproduction. Similairement, pour Les éphémères, les cadrages laissaient souvent voir les pousseurs de chariots, pour la plupart eux-mêmes acteurs et actrices du spectacle.

Ces velléités de monstration du théâtre constituent une manifestation récurrente du travail de Mnouchkine. Durant la présentation sur scène du Dernier caravansérail, l'espace de la frontière est physiquement symbolisé par la rampe du théâtre elle-même - cet espace est visuellement renforcé par une clôture grillagée. Matériellement, et pour les besoins du récit, la rampe maintient donc sa fonction de division entre deux espaces et s'inscrit symboliquement comme limite frontalière diégétique. Lors de la performance en public, comme pour celle des Éphémères, ce sont les machinistes qui font tourner les décors afin d'offrir diverses perspectives à l'audience. En revanche, pour le tournage du Dernier caravansérail, c'est la caméra qui contourne et pénètre les décors, décuplant les points de vue; la caméra adopte une stratégie similaire pour la plupart des séquences du film. Or, contrairement à tout autre moment où le spectateur de l'écran est invité à l'intérieur des décors ou aux côtés des personnages sur le plateau, les scènes de passage des frontières sont filmées d'un point de vue frontal et souvent statique, à l'instar de celui de la spectatrice de la salle. Pourquoi, alors que Mnouchkine mobilise les procédés filmiques pour faire oublier la rampe tout au long du film, briser cette illusion durant les scènes de passage des frontières à intervalles réguliers? D’autant plus que Le dernier caravansérail 
s'évertue sous maints aspects à masquer la matérialité du théâtre en exploitant les ressources de l'illusion que lui procure le support filmique afin de réduire l'écart entre la scène et l'écran ${ }^{29}$.

À ce propos, dans la dernière note de son article « Réflexions sur la réflexivité théâtrale ${ }^{30} »$, Jean-Claude Vuillemin écrit qu' «il est théoriquement impossible de faire l'économie [de la métathéâtralité] ${ }^{31}$ ». En effet, Vuillemin pose «comme axiomatique que représenter implique l'exhibition inéluctable de la dimension autotélique du processus » théâtral et que « le théâtre ne saurait se représenter sans se représenter lui-même représentant. Sans évoquer, ne serait-ce que de manière détournée ou implicite, son propre statut, ses propres procédés de création et de réception ${ }^{32} \gg$. La métathéâtralité détermine le théâtre et le constitue simultanément au sein d'une dialectique de transitivité et de référentialité. Ainsi le théâtre se regardet-il autant, sinon plus, qu'il donne à voir.

Or, si le «signe-théâtre » le plus immédiatement identifiable dans ce film reste incontestablement la rampe et que, comme le dit Vuillemin, « indispensable à la représentation, [la rampe] ne peut faire autrement que $\mathrm{d}[$ e s]'exhiber [...] en tant que telle ${ }^{33} \gg$, alors l'exposition récurrente de la rampe fait surtout émerger la nature particulière du film-théâtre. Les scènes de passage des frontières soulignent ainsi moins une manière pour le théâtre de rivaliser avec le cinéma que d'exiger sa place dans le film, car la distinction esthétique récurrente qui marque ces moments souligne une rupture non de l'illusion fictionnelle - le récit ne s'interrompt pas -, mais de l'ontologie filmique afin de réintroduire les procédés du théâtre. Alors que son absence du cadre de l'objectif durant la majorité des scènes favorise l'illusion filmique, la rampe réapparaît en effet de manière régulière, à la manière d'une anaphore, figure rythmique qui rappelle formellement que c'est le théâtre qui est à l'œuvre, et non un film de fiction - les retours épisodiques au théâtre sont d'autant plus manifestes que les moyens déployés pour faire oublier que l'action prend place sur une scène sont puissants.

${ }^{29}$ Je suggère aux lectrices de visionner la scène ayant lieu dans une cabine téléphonique au bord de la Tamise, afin qu'elles se rendent compte du véritable coup de théâtre ontologique que réussit Mnouchkine.

${ }^{30}$ Jean-Claude Vuillemin, « Réflexions sur la réflexivité théâtrale », L'Annuaire théâtral, $\mathrm{n}^{\circ}$ 45, printemps 2009, p. II9-35, https://id.erudit.org/iderudit/044277ar (consulté le 23 mars 2019).

${ }^{31}$ Ibid., p. 133.

${ }^{32}$ Ibid., p. 126.

33 Ibid., p. I30. 
Si les scènes de passage des frontières du Dernier caravansérail soulignent une tendance du théâtre à s'exhiber comme tel, la subjectivité des points de vue des personnages dans Les éphémères est également exprimée selon une rhétorique filmique qui n'est pas celle des codes cinématographiques d'un film de fiction, mais qui, à l'instar du Bal, prend en compte la spatialité scénique. Lorsque la femme de «Chez Gaëlle » regarde la petite fille sur un chariot surgi des coulisses, le découpage du film s'effectue selon un va-et-vient entre les deux personnages afin de suggérer le lien temporel qui les unit - c'est elle-même jeune fille qu'elle regarde. Cependant, alors que les conventions cinématographiques prescrivent des prises de vue subjectives dans une situation similaire afin de favoriser l'identification de l'instance spectatrice aux personnages de la diégèse, la caméra de Mnouchkine n'adopte techniquement le point de vue d'aucune des comédiennes. Dans le cinéma traditionnel, l'utilisation du champ/contrechamp est devenue systématique dans les séquences de dialogue : le personnage qui parle est visible à l'intérieur des limites de l'écran, tandis que celui à qui il s'adresse reste en hors-champ — dans certains cas, une fraction du corps de l'interlocuteur demeure dans le cadre. Cette méthode de filmage des dialogues peut acquérir une fonction symbolique puisque, d'un point de vue sémiotique, les personnages sont isolés: dans la scène de «Chez Gaëlle », en l'occurrence, cette séparation incarne la distance temporelle dissociant les deux moments de la vie du personnage. Filmer un dialogue en incluant les personnages dans un même plan génère une autre interprétation, puisqu'un tel partage de l'espace suscite une impression d'entente - dans cette scène, les deux Gaëlle se trouvent finalement cadrées ensemble.

Mnouchkine favorise donc des méthodes de filmage distinctes afin de susciter la subjectivité. En effet, l'identification aux personnages opère à deux niveaux dans un film de fiction. D'une part, la focalisation interne sollicite l'objectivation $\mathrm{du}$ subjectif car, pour que le spectateur puisse s'identifier à un personnage, il faut que le premier voie le second ${ }^{34}$. L'identification subjective à un personnage nécessite la connaissance objective de ce personnage, phénomène paradoxal que souligne Christian Metz: «De telles images subjectives supposent, pour être correctement comprises, que des images objectives montrant le héros lui-même soient présentées dans le film [...]; le spectateur, en effet, ne peut s'identifier au point de vue du héros

${ }^{34}$ Contrairement à ce que tentait de prouver Robert Montgomery avec son film de 1947, Lady in the Lake. 
que s'il connaît ce héros ${ }^{35}$. » Or, pour le tournage des Éphémères, le processus d'identification s'accomplit également dans un paradoxe, bien que celui-ci soit de nature différente : la subjectivité est effectivement créée à l'aide du montage, alternant des plans rapprochés de l'une et de l'autre Gaëlle, mais la caméra reste en retrait, et n'adopte jamais le point de vue physique ou optique du personnage. Narrativement, le point de vue est subjectif et appartient à Gaëlle adulte, mais, optiquement, il reste objectif; il est celui du technicien derrière la caméra ou celui, multiple, du public de la salle. Alors que Mnouchkine exploite l'ubiquité essentielle au film de fiction afin d'offrir une multiplicité de perspectives à laquelle l'audience de la salle n'a pas souvent accès, la cinéaste n'autorise pas pour autant l'accès physique à la subjectivité des personnages - le point de vue est communiqué de manière davantage narrative pendant la présentation scénique et grâce au montage dans le film. Mnouchkine produit ainsi une focalisation interne à l'aide de procédés étrangers au médium cinématographique, qui prennent en considération les conventions scéniques et sont par conséquent mieux adaptés à l'esthétique du film-théâtre.

Ces trois exemples permettent d'éclairer la singularité de l'énonciation hybride caractérisant le régime de présentation du film-théâtre, un tiers mode de présentation dramatique né de la rencontre entre les caractéristiques d'un événement scénique et de codes filmiques. La version filmique du Bal prend en compte les particularités spatiotemporelles du théâtre, celle du Dernier caravansérail s'articule autour de la métathéâtralité constitutive du processus scénique, tandis que Les éphémères évoquent la subjectivité de manière extrinsèque à la rhétorique cinématographique conventionnelle. Ce phénomène d'influence réciproque démontre que non seulement l'échange intermédial contribue à l'enrichissement de chaque média, mais surtout qu'il concourt à mieux définir la nature du film-théâtre.

En outre, et ainsi que le signale la catégorie de « références intermédiales » définie par Rajewsky, le film-théâtre, puisqu'il fait référence à un autre média, n’est pas un événement autonome. Or, bien qu'il assimile certaines particularités scéniques, il n'est pas pour autant asservi aux conventions de présentation de l'événement qu'il filme. Si son énonciation met au jour des liens de dépendance lui interdisant de s'autonomiser tout à fait, il continue de cultiver des relations avec le mode d'expression filmique. Son énonciation se singularise donc au sein d'une double tension: il n'imite pas l'événement qu'il filme, mais s'approprie certains procédés scéniques qu'il ajuste alors à son domaine. D’une part, cette tension crée un

\footnotetext{
${ }_{35}$ Christian Metz, Essais sur la signification au cinéma, tome 2, Paris, Klincksieck, 1972, p. $45-46$.
} 
espace de singularité pour l'énonciation du film-théâtre, comme en témoignent certains choix de réalisation. Mais de l'autre, cette tension lui interdit en même temps de se distancier complètement des productions le précédant: le plan d'ensemble privilégié par Ettore Scola pour Lebal évoque la spatialité de la scène; la multiplication de plans immotivés d'objets dans Lacantatrice chauve de Vincent Bataillon renvoie à l'incohérence d'abord mise en scène par Jean-Luc Lagarce et à la thématique exprimée par Ionesco avant lui; Mnouchkine prend en compte la machinerie du théâtre, puisque les acteurs et les actrices qui participent aux changements de décor restent inclus dans le cadre. Ces liens d'interdépendance soulignent donc une complicité entre cinéma et théâtre qui, paradoxalement, met en lumière l'indépendance fondamentale de ces films.

Bien qu'André Bazin ${ }^{36}$ ait, dès 1951, estimé que le matériau scénique résistait à se laisser transposer sur support filmique en raison de leur système signifiant hétérogène, nombre de constats sur l'irréductible porosité générique ont depuis vu le jour. Jacques Derrida, avec son article de 1980, « La loi du genre ${ }^{37} »$, a déconstruit la notion de classification : « un texte ne saurait appartenir à aucun genre. Tout texte participe d'un ou de plusieurs genres, il n'y a pas de texte sans genre, il y a toujours du genre et des genres, mais cette participation n'est jamais une appartenance ${ }^{38} \gg$. Cette loi du « débordement ${ }^{39}$ » souligne un fait paradoxal, puisque $d u$ genre s'établit à partir d'un élément étranger à sa propre catégorie ${ }^{40}$. En 2004, Marvin Carlson souligne encore ce phénomène lorsqu'il cite Trinh T. Minh-Ha dans la conclusion de Performance : a critical introduction: «despite our desperate, eternal attempt to separate, contain and mend, categories always leak ${ }^{4 \mathrm{I}} \gg$. Ces postulats prêtent à des réflexions concernant les processus à l'œuvre lors d'interactions médiatiques.

\footnotetext{
${ }^{36}$ Bazin, I95I, p. 89I-905.

37 Jacques Derrida, « La loi du genre », Glyph 7 (1980), p. 176-20I.

${ }^{38} \mathrm{Ibid}$, p. 185.

39 Ibid, p. 184.

$4^{\circ}$ Frédérique Toudoire-Surlapierre, «Derrida, Blanchot, "Peut-être l'extase" », « Les philosophes lecteurs », Fabula LHT (Littérature, histoire, théorie), n I, février 2006, http://www.fabula.org/lht/I/Toudoire-Surlapierre.html (consulté le 20 mars 2019). L'autrice note que, si « l'appartenance relève plus du don que de la propriété, elle pose davantage la question de "l'identification" (c'est-à-dire du genre auquel elle se rapporte) que celle de l'identité » (paragraphe 5). Son raisonnement soulève une hypothèse importante pour la définition du film-théâtre : libéré de contraintes d'inclusion ou d'exclusion, il se constitue au sein d'une inévitable perméabilité entre des catégories limitrophes.

${ }^{4 \mathrm{I}}$ Marvin Carlson, Performance: a critical introduction, London, Routledge, 1996, p. 190.
} 
Gaudreault et Marion notent ainsi que « a good understanding of a medium [...] entails understanding its relationship to other media: it is through intermediality, through a concern with the intermedial, that a medium is understood ${ }^{42} \gg$; Sybille Kramer, que : «intermediality is an epistemological condition of mediarecognition ${ }^{43} » ;$ et, enfin, Jürgen E. Müller, qu' « un média recèle en soi des structures et des possibilités qui ne lui appartiennent pas exclusivement ${ }^{44} \gg$, que cette « complicité conceptuelle [possède des] stratifications esthétiques [qui] ouvrent d'autres voies à l'expérience $45 \gg$.

Ce contexte d'interpénétration médiatique ouvre ainsi de nouvelles voies pour l'appréciation «monumentale » du film-théâtre. Déjà exploité à des fins de « document », en tant qu'objet référentiel, puisqu'il constitue un témoignage tangible pour les praticiens, chercheurs et enseignants du théâtre et du cinéma, ou comme référence pour d'éventuelles interprétations futures, il est désormais en mesure de s'affranchir de références à une quelconque origine, de se « monumentaliser », au sens foucaldien du terme. La mise en scène de Lagarce agit comme « document » lorsque ce dernier met en scène les commentaires que Ionesco avait ajoutés aux éditions subséquentes de son texte sur le travail de Nicolas Bataille, indiqués en notes de bas de pages et déclamés par la bonne. De même, la réalisation de Vincent Bataillon documente l'absurdité consensuelle du texte dramatique et l'inspiration télévisuelle du texte scénique de Lagarce, mais le film, aboutissement de cette activité intermédiale, revendique surtout sa propre énonciation et postule par conséquent une exégèse « monumentale ».

Didier Plassard avait examiné deux mises en scène de Dom Juan : celle de Jean Vilar, le 15 juillet 1953 dans la Cour d'honneur du Palais des papes à Avignon, et celle de Jean Meyer, le 5 novembre 1952 à la Comédie-Française. Il avait noté que les comptes rendus de la presse confirmaient une préférence pour la création de Vilar, plutôt que pour la lecture renouvelée de Meyer. Les réactions du public face aux deux interprétations du texte dramatique de Molière soulignaient ainsi un changement

\footnotetext{
${ }^{42}$ André Gaudreault et Philippe Marion, “The Cinema as a Model for the Genealogy of Media,” Convergence, vol. 8, $\mathrm{n}^{\circ} 4, \quad$ December 2002, p. 15-16, https://journals.sagepub.com/doi/abs/Io.I177/135485650200800402 (consulté le I4 août 20I9).

43 Rajewsky, 2005, p. 48.

${ }^{44}$ Müller, 200o, p. I.

45 Ibid., p. II3.
} 
important, à savoir que la création de Vilar n'entrait pas en conflit avec la transmission de l'œuvre de Molière. Plassard concluait alors que

l'archive et l'invention [...] ne sauraient s'opposer. La quête d'appuis documentaires et l'appel à des modes de figuration inédits sont les deux procédures complémentaires par lesquelles la création théâtrale, depuis la naissance de la mise en scène, s'écarte de la transmission mémorielle pour se risquer dans l'aventure d'une exploration sans cesse renouvelée ${ }^{46}$.

Ainsi, le film-théâtre prouve qu'il n'est plus réduit à sa seule fonction d'objet d'archive, de «document ». Si l'objectif initial de l'enregistrement audiovisuel l'identifiait à la conservation du texte scénique, la conversion de l'archive filmique en œuvre n'est plus impensable. Les deux activités de préservation et de création ne s'opposent pas, elles coexistent. Le support filmique remplit une fonction de protection du texte scénique, parfois à des fins de transmission, car il lui confère une pérennité qui ne lui a pas toujours été permise et une empiricité qui en facilite l'accès. Le film-théâtre continue donc de remplir une mission utilitaire du patrimoine théâtral, en parallèle avec les carnets des dramaturges, les photos, les dessins, etc. Cependant, sa relation intermédiale avec la scène confère au film-théâtre une qualité créatrice car, dès qu'il transmet le texte scénique, il en prolonge la lecture. Interprète, il constitue un nouvel objet intermédial, dont la double fonction syncrétique, « documentaire » et « monumentale », lui confère une valeur créatrice dès que les images du texte scénique sont mises en scène par le réalisateur. Le film-théâtre est donc fondateur, puisqu'il n'est pas seulement créateur de son propre texte, mais parce que de manière rétroactive, il offre une lecture inédite du texte scénique ${ }^{47}$. Ainsi

${ }^{46}$ Didier Plassard, « La scène peut-elle être un lieu de mémoire ? », Vincent Amiel et Gérard-Denis Farcy (dir.), Mémoire en éveil, archives en création. Le point de vue du théatre et du cinéma, Vic la Gardiole, L'Entretemps, 2006, p. 26. L'auteur s'appuie sur la distinction effectuée par Pierre Nora entre «mémoire » et « histoire » dans Lieux de mémoire afin d'envisager l'archive comme moyen d'aménager une voie d'accès au passé (histoire) et comme lien avec le présent (mémoire) : « les lieux de mémoire ne sont pas ce dont on se souvient, mais là où la mémoire travaille; non la tradition elle-même, mais son laboratoire » (Pierre Nora, Les lieux de mémoire, Paris, Gallimard, «Bibliothèque illustrée des histoires », tome I, 1984, p. 26). Ces mises en scène sont donc valorisées autant pour leur « histoire » en tant que « documents » que pour leur « mémoire » en tant que « monuments ».

47 Ce même phénomène pourrait décrire un lien similaire entre texte scénique et texte dramatique, dont le film-théâtre se porte garant de la (ré)écriture en vertu de sa capacité de préservation immuable. 
l'analyse du film de Bataillon a-t-elle mis au jour les manières dont il s'est réapproprié l'absurdité visible en filigrane sur le palimpseste de La cantatrice chauve, tout comme Lagarce avait renouvelé sa propre mise en scène avec des emprunts faits à l'esthétique télévisuelle dans l'objectif, si ce n'est ludique, d'amplifier et de poursuivre les thématiques à l'œuvre dans le texte de Ionesco.

De ce fait, et peut-être à l'instar des auteurs que Michel Foucault a qualifiés d'« instaurateurs de discursivité », le film-théâtre produit « quelque chose de plus : la possibilité et la règle de formation d'autres textes $4^{4} \gg$. En ce sens, et comme les auteurs dont la pensée a creusé un espace discursif, condition de possibilités infinies d'autres discours hétérogènes aux leurs, mais qui pourtant en découlent et les déploient, le film-théâtre relance et enrichit la lecture de textes produits avant lui. Car, ce qui caractérise l'instauration de discursivité, c'est creuser ce qui est dit à travers le texte « d'origine », et non pas redire la même chose. Le « retour à l'origine » selon Foucault,

fait partie du discours lui-même, [...] il n'est pas un supplément historique qui viendrait s'ajouter à la discursivité elle-même et la redoublerait d'un ornement qui, après tout, n'est pas essentiel; il est un travail effectif et nécessaire de transformation de la discursivité elle-même [...] ce qui manifeste l'acte [d'instauration], ce qui en dérive, c'est, en même temps, ce qui établit l'écart et ce qui le travestit ${ }^{49}$.

Le film-théâtre constitue par conséquent un événement intermédial qui propose une lecture à la fois documentaire et monumentale — « document » et « monument » constituent deux facettes consubstantielles du film-théâtre, et c'est la manière de le considérer qui conditionne son rôle documentaire et/ou monumental. La capacité du film-théâtre comme moyen de sauvegarde idéal du texte spectaculaire l'avait d'abord cantonné à ses fonctions de préservation, de transmission et de réactivation mémorielle, lui interdisant toute ambition artistique. Du fait de sa capacité de preuve, d'ancrage dans le réel, puisque restituant le temps, le son et l'espace de manière synchrone, il offre effectivement un avantage quant à sa capacité de documentation du théâtre, d'analyse du processus créatif, et constitue une mémoire précieuse pour les archives du spectacle.

${ }^{48}$ Michel Foucault, «Qu'est-ce qu'un auteur ? », L'archéologie du savoir, Paris, Gallimard, 1969, p. 804.

49 Ibid., p. 8 o8. 
I33 Néanmoins, affranchi de son devoir de «document », le film-théâtre acquiert un statut en tant que pratique et événement autotéliques que le concept d'intermédialité a permis de valider en soulignant une interdépendance mutuelle et constante entre la scène et l'écran.

Les diverses itérations de La cantatrice chauve témoignent assurément de cette complicité intermédiale. Nombre de metteurs en scène s'approprient certaines caractéristiques filmiques et les accordent à leurs propres dispositifs scéniques afin de contourner les contraintes du tournage avec un public in situ, d'imiter la simultanéité spatiotemporelle, d'augmenter les possibilités focalisatrices de leur public ou, à l'instar de Lagarce, de perpétuer en l'actualisant une thématique exprimée au préalable.

En revanche, les analyses du Bal, des Éphémères et du Derniercaravansérail ont démontré l'influence des pratiques scéniques sur l'esthétique du film-théâtre : les cinéastes évitent les coupures de montage - permettant encore de recréer un continuum spatiotemporel - , choisissent de conserver une perspective optiquement objective pour créer un effet de subjectivité, ou encore prennent en compte la machinerie du théâtre en incluant dans le champ de la caméra les acteurs et les actrices qui participent aux changements de décor. Le processus de filmage s'écarte ainsi de procédés qui renvoient aux factures cinématographiques conventionnelles, pour les ajuster sur le plan formel du discours filmique; ce que réussit Bataillon lorsqu'il éclaire la nature du lien intermédial entre trois pratiques - littéraire, spectaculaire et filmique - pour illustrer la manière dont le film reprend à son compte l'idéologème $e^{\text {so }}$ de l'absurde.

De ce fait, si la pratique scénique ne peut plus ignorer les spécificités filmiques ni se concevoir sans emprunter à celles-ci, il est manifeste que ce phénomène d'influence réciproque s'avère fondateur pour le film-théâtre. Comme le précise Rajewsky, les manières dont un média utilise ses propres moyens d'expression pour faire référence à un autre média entraînent une transformation du premier, le média de référence, matériellement présent. Ce sont donc les manières dont il négocie ce passage intermédial qui ont permis de créer un espace de singularité d'où peut émerger l'esthétique inédite du film-théâtre.

so Néologisme de Julia Kristeva : articulation d'un texte sur d'autres structures, « Le Texte clos », Langages, n I2, décembre 1968, p. I04, https://www.persee.fr/doc/lgge_0458726x_1968_num_3_I2_2356 (consulté le 29 juillet 2019). 


\title{
Film-théâtre, intermédialité et nouveaux enjeux esthétiques
}

\author{
Sandrine Siméon, Soka University of America
}

\section{RÉSUMÉ}

Comment l'analyse intermédiale permet-elle de souligner l'interpénétration médiatique à l'œuvre entre la performance scénique et les modes d'écriture de l'enregistrement audiovisuel du spectacle vivant ? Cet article évalue les manières dont la rhétorique filmique agit sur le dispositif scénique, puis comment, à son tour, la scène influence l'esthétique inédite du film-théâtre, lui conférant un statut autotélique.

\section{ABSTRACT}

In what ways does intermedial analysis highlight the interactions that occur between media when live performances are being recorded? This article explores how film rhetoric influences theatrical staging and, in turn, how the scenographic apparatus affects the innovative aesthetic of film-theatre, granting it autotelic status.

\section{NOTE BIOGRAPHIQUE}

SANDRINE SIMÉON Est professeure adjointe à la Soka University, aux États-Unis, et y enseigne la langue, la culture et le cinéma français et francophones. Sa recherche se focalise sur le filmage de la scène, et ses publications incluent des articles pour European Drama and Performance Studies (vol. 3, n 3, 20I4), Romance Studies (vol. 35, $\mathrm{n}^{\circ} 4,2017$ ), Brasilian Journal on Presence Studies (vol. 7, $\mathrm{n}^{\circ}$ 3, 2017), Théâtre, levain du cinéma ; théâtre, destin du cinéma (2013), et un volume pour la revue du Radac, Coup de théâtre ( $\left.\mathrm{n}^{\circ} 3 \mathrm{I}, 2017\right)$. 